\title{
Subgroups of the Group of Generalized Lorentz Transformations and Their Geometric Invariants
}

\author{
George Yu. BOGOSLOVSKY
}

Skobeltsyn Institute of Nuclear Physics, Moscow State University, 119992 Moscow, Russia

E-mail: bogoslov@theory.sinp.msu.ru

Received October 06, 2005, in final form November 09, 2005; Published online November 15, 2005

Original article is available at http://www.emis.de/journals/SIGMA/2005/Paper017/

\begin{abstract}
It is shown that the group of generalized Lorentz transformations serves as relativistic symmetry group of a flat Finslerian event space. Being the generalization of Minkowski space, the Finslerian event space arises from the spontaneous breaking of initial gauge symmetry and from the formation of anisotropic fermion-antifermion condensate. The principle of generalized Lorentz invariance enables exact taking into account the influence of condensate on the dynamics of fundamental fields. In particular, the corresponding generalized Dirac equation turns out to be nonlinear. We have found two noncompact subgroups of the group of generalized Lorentz symmetry and their geometric invariants. These subgroups play a key role in constructing exact solutions of such equation.
\end{abstract}

Key words: Lorentz, Poincaré and gauge invariance; spontaneous symmetry breaking; Finslerian space-time

2000 Mathematics Subject Classification: 53C60; 53C80

\section{Introduction}

It is common knowledge that since its discovery and for a hundred years ahead the Lorentz symmetry has determined the development of the theory of fundamental interactions. At present, however, owing to progress made in the construction of unified gauge theories, there have been grounds to consider the Lorentz symmetry as not strict but only approximate symmetry of nature.

According to the most popular point of view, even in a theory which has Lorentz invariance at the fundamental level, this symmetry can be spontaneously broken if some (for example, vector) field acquires a vacuum expectation value which breaks the initial Lorentz symmetry. Certainly the question here concerns symmetry violation with respect to active Lorentz transformations of fundamental fields against the background of fixed (in this case, vector) condensate. As to the passive Lorentz transformations under which the condensate is transformed as a Lorentz vector, the corresponding Lorentz covariance remains valid.

Noteworthy is the fact that the usual Standard Model of strong, weak and electromagnetic interactions does not have the dynamics necessary to cause spontaneous breaking of the Lorentz symmetry. In other words, the standard Higgs mechanism, which breaks the local gauge symmetry and gives rise to the scalar Higgs condensate, does not affect the initial Lorentz symmetry of the theory.

In order to describe possible effects caused by violation of active Lorentz invariance and to classify them as effects of Planck-scale physics, strongly suppressed at attainable energy scales, the so-called Standard Model Extension has been proposed [1, 2, 3, 4]. The Lagrangian of this phenomenological theory is constructed so that it includes all passive Lorentz scalars formed by combining standard-model fields with coupling coefficients having Lorentz indices. As a result, along with the Lorentz symmetry the relativistic symmetry turns out to be ad-hoc violated. 
In contrast to such string-motivated theory (see also $[5,6]$ ) there exists another, Finslerian approach to the problem $[7,8,9,10]$, which permits Lorentz symmetry violation without violation of relativistic symmetry. It is based on the following idea. Spontaneous breaking of the initial gauge symmetry may be accompanied by the corresponding phase transition in the geometrical structure of space-time. In other words, spontaneous breaking of the gauge symmetry may lead to a dynamic rearrangement of the vacuum which results in the formation of a relativistically invariant anisotropic fermion-antifermion condensate, i.e. of a constant classical nonscalar field ${ }^{1}$. This constant field physically manifests itself as a relativistically invariant anisotropic medium filling space-time. Such a medium, leaving space-time flat, gives rise to its anisotropy, that is, instead of Minkowski space, there appears a relativistically invariant Finslerian event space with partially broken 3D isotropy.

\section{The group of isometries of the flat Finslerian space-time with partially broken 3D isotropy}

In comparison with the 6-parameter homogeneous Lorentz group of Minkowski space, the homogeneous group of isometries of the flat Finslerian space with partially broken 3D isotropy is a 4-parameter group. Apart from 3-parameter boosts (generalized Lorentz transformations) it includes only axial symmetry transformations, i.e. the 1-parameter group of rotations around the preferred direction in 3D space, in which case this direction is determined by a spontaneously arising axially symmetric fermion-antifermion condensate. It will be demonstrated below that the 4-parameter group of Finslerian isometries is locally isomorphic to the corresponding 4-parameter subgroup of the Lorentz group. Therefore we primarily consider the 4-parameter subgroup of the Lorentz group.

\subsection{The 4-parameter subgroup of Lorentz group and its 3-parameter noncompact subgroup}

In [12], in terms of Lie algebras all continuous subgroups of the Lorentz group were classified. It turned out that the Lorentz group contains no 5-parameter subgroups and has only one (up to isomorphism) 4-parameter subgroup. This subgroup includes independent rotations around an arbitrarily selected axis, the direction of which will be denoted using a unit vector $\boldsymbol{\nu}$, and a 3-parameter group consisting of noncompact transformations only. Physically such noncompact transformations are realized as follows. First choose as $\boldsymbol{\nu}$ a direction towards a preselected star and then perform an arbitrary Lorentz boost and complement it with such a turn of the spatial axes that in a new reference frame the direction towards the star remains unchanged.

The set of the transformations described, while linking the inertial reference frames, actually constitutes a 3-parameter noncompact group (in contrast to the usual Lorentz boosts). Let us write the corresponding 3-parameter transformations in the infinitesimal form

$$
\begin{aligned}
& d x^{0}=-\boldsymbol{n x} d \alpha, \\
& d \boldsymbol{x}=\left(-\boldsymbol{n} x^{0}-[\boldsymbol{x}[\boldsymbol{\nu} \boldsymbol{n}]]\right) d \alpha,
\end{aligned}
$$

where $d \alpha$ is a rapidity, the unit vector $\boldsymbol{n}$ indicates a direction of the infinitesimal boost, so that $d \boldsymbol{v}=\boldsymbol{n} d \alpha$, and the meaning of $\boldsymbol{\nu}$ has been explained ${ }^{2}$. Integration of equations (1) leads to the

\footnotetext{
${ }^{1}$ It is worth noting that, irrespective of the problem of violation of Lorentz symmetry, in the literature consideration has already been given to the mechanism of the dynamical breaking of the initial gauge symmetry which is alternative to the standard one; instead of the elementary Higgs condensate there appears a scalar fermion-antifermion condensate [11].

${ }^{2}$ For more details see [7].
} 
finite transformations which, at any fixed $\boldsymbol{\nu}$, belong to the Lorentz group and themselves form a 3-parameter noncompact group with parameters $\boldsymbol{n}, \alpha$ :

$$
x^{\prime} i=\Lambda_{k}^{i}(\boldsymbol{\nu} ; \boldsymbol{n}, \alpha) x^{k},
$$

where

$$
\begin{aligned}
& \Lambda_{0}^{0}=1+\frac{\cosh \boldsymbol{\nu} \boldsymbol{n} \alpha-1}{(\boldsymbol{\nu} \boldsymbol{n})^{2}}, \\
& \Lambda_{\beta}^{0}=\frac{1-e^{-\boldsymbol{\nu} \boldsymbol{n} \alpha}}{\boldsymbol{\nu} \boldsymbol{n}} n_{\beta}+\frac{\cosh \boldsymbol{\nu} \boldsymbol{n} \alpha-1}{(\boldsymbol{\nu} \boldsymbol{n})^{2}} \nu_{\beta}, \\
& \Lambda_{0}^{\rho}=\frac{1-e^{\boldsymbol{\nu} \boldsymbol{n} \alpha}}{\boldsymbol{\nu} \boldsymbol{n}} n^{\rho}+\frac{\cosh \boldsymbol{\nu} \boldsymbol{n} \alpha-1}{(\boldsymbol{\nu} \boldsymbol{n})^{2}} \nu^{\rho}, \\
& \Lambda_{\beta}^{\rho}=\delta_{\beta}^{\rho}+\frac{1-e^{\boldsymbol{\nu} \boldsymbol{n} \alpha}}{\boldsymbol{\nu} \boldsymbol{n}} n^{\rho} \nu_{\beta}+\nu^{\rho} \Lambda_{\beta}^{0} .
\end{aligned}
$$

Hereafter the Latin indices take on values of $0,1,2,3$ while the Greek ones, values of $1,2,3$. Note also that the $n^{\beta}$ and $\nu^{\beta}$ denote the Cartesian components of unit vectors $\boldsymbol{n}$ and $\boldsymbol{\nu}$, in which case $n_{\beta}=-n^{\beta}, \nu_{\beta}=-\nu^{\beta}$. The transformations inverse to (2) appear as

$$
x^{i}=\Lambda_{k}^{-1_{k}^{i}}(\boldsymbol{\nu} ; \boldsymbol{n}, \alpha) x^{\prime k},
$$

where

$$
\Lambda_{k}^{-1_{k}^{i}}(\boldsymbol{\nu} ; \boldsymbol{n}, \alpha)=\Lambda_{k}^{i}(\boldsymbol{\nu} ; \boldsymbol{n},-\alpha) .
$$

Consider two arbitrary elements of the group (2). Let the first element $g_{1}$ be characterized by the parameters $\boldsymbol{n}_{1}, \alpha_{1}$, and the second one, $g_{2}$, by the parameters $\boldsymbol{n}_{2}, \alpha_{2}$. Then the element $g=g_{2} g_{1}$ will have the corresponding parameters $\boldsymbol{n}, \alpha$, which are functionally dependent on $\boldsymbol{n}_{1}, \alpha_{1}$ and $\boldsymbol{n}_{2}, \alpha_{2}$, i.e. $\Lambda_{k}^{i}(\boldsymbol{\nu} ; \boldsymbol{n}, \alpha)=\Lambda_{j}^{i}\left(\boldsymbol{\nu} ; \boldsymbol{n}_{2}, \alpha_{2}\right) \Lambda_{k}^{j}\left(\boldsymbol{\nu} ; \boldsymbol{n}_{1}, \alpha_{1}\right)$. Using the explicit form of the matrix elements $\Lambda_{k}^{i}(\boldsymbol{\nu} ; \boldsymbol{n}, \boldsymbol{\alpha})$ and making the relevant calculations, we arrive at the following relations:

$$
\begin{aligned}
& \boldsymbol{n} \alpha=\frac{\boldsymbol{\nu}\left(\boldsymbol{n}_{1} \alpha_{1}+\boldsymbol{n}_{2} \alpha_{2}\right)}{1-e^{\boldsymbol{\nu}\left(\boldsymbol{n}_{1} \alpha_{1}+\boldsymbol{n}_{2} \alpha_{2}\right)}}\left[\frac{1-e^{\boldsymbol{\nu} \boldsymbol{n}_{1} \alpha_{1}}}{\boldsymbol{\nu} \boldsymbol{n}_{1}} \boldsymbol{n}_{1}+\frac{e^{\boldsymbol{\nu} \boldsymbol{n}_{1} \alpha_{1}}\left(1-e^{\boldsymbol{\nu} \boldsymbol{n}_{2} \alpha_{2}}\right)}{\boldsymbol{\nu} \boldsymbol{n}_{2}} \boldsymbol{n}_{2}\right], \\
& \boldsymbol{n}^{2}=1 .
\end{aligned}
$$

These relations essentially represent the law of group composition for the 3-parameter noncompact subgroup (2) of the Lorentz group.

Since the group (2) links the coordinates of events in the initial and primed inertial reference frames, from the physical standpoint it is more natural to use as group parameters three velocity components, $\boldsymbol{v}$, of the primed reference frame rather than the $\boldsymbol{n}, \alpha$. In order to express the $\boldsymbol{v}$ in terms of the $\boldsymbol{n}, \alpha$ it is sufficient to consider in the initial frame, the motion of the origin of the primed frame, i.e. to write $x^{\beta}=\Lambda^{-1}{ }_{0}^{\beta} x^{\prime 0}$ and $x^{0}=\Lambda^{-1}{ }_{0}^{0} x^{\prime 0}$. Then $v^{\beta}=x^{\beta} / x^{0}=\Lambda^{-1}{ }_{0}^{\beta} / \Lambda^{-1}{ }_{0}^{0}$. Using equations (2)-(4), we get as a result

$$
\boldsymbol{v}=\left[\frac{1-e^{-\boldsymbol{\nu} \boldsymbol{n} \alpha}}{\boldsymbol{\nu} \boldsymbol{n}} \boldsymbol{n}+\frac{\cosh \boldsymbol{\nu} \boldsymbol{n} \alpha-1}{(\boldsymbol{\nu} \boldsymbol{n})^{2}} \boldsymbol{\nu}\right] /\left[1+\frac{\cosh \boldsymbol{\nu} \boldsymbol{n} \alpha-1}{(\boldsymbol{\nu} \boldsymbol{n})^{2}}\right] .
$$

Hereafter we put $c=1$. Since $\boldsymbol{n}^{2}=\boldsymbol{\nu}^{2}=1$, equation (7) yields the inverse relations:

$$
\begin{aligned}
& \boldsymbol{n}=\frac{\boldsymbol{v}}{\sqrt{2(1-\boldsymbol{v} \boldsymbol{\nu})\left(1-\sqrt{1-\boldsymbol{v}^{2}}\right)}}-\sqrt{\frac{1-\sqrt{1-\boldsymbol{v}^{2}}}{2(1-\boldsymbol{v} \boldsymbol{\nu})}} \boldsymbol{\nu} \\
& \alpha=\frac{\sqrt{2(1-\boldsymbol{v} \boldsymbol{\nu})\left(1-\sqrt{1-\boldsymbol{v}^{2}}\right)}}{\sqrt{1-\boldsymbol{v}^{2}}+\boldsymbol{v} \boldsymbol{\nu}-1} \ln \left(\frac{\sqrt{1-\boldsymbol{v}^{2}}}{1-\boldsymbol{v} \boldsymbol{\nu}}\right) .
\end{aligned}
$$


In terms of $\boldsymbol{v}$ the law of group composition (5), (6) takes the form

$$
\boldsymbol{v}=\frac{\left(\boldsymbol{v}_{1}\left(1-\boldsymbol{v}_{2} \boldsymbol{\nu}\right)+\boldsymbol{v}_{2} \sqrt{1-\boldsymbol{v}_{1}^{2}}\right)\left(1-\boldsymbol{v}_{1} \boldsymbol{\nu}\right)+\boldsymbol{\nu}\left(\boldsymbol{v}_{1} \boldsymbol{v}_{2}+\boldsymbol{\nu} \boldsymbol{v}_{2}\left(\sqrt{1-\boldsymbol{v}_{1}^{2}}-1\right)\right) \sqrt{1-\boldsymbol{v}_{1}^{2}}}{1-\boldsymbol{v}_{1} \boldsymbol{\nu}+\boldsymbol{v}_{1} \boldsymbol{v}_{2} \sqrt{1-\boldsymbol{v}_{1}^{2}}+\boldsymbol{\nu} \boldsymbol{v}_{2}\left(1-\boldsymbol{v}_{1} \boldsymbol{\nu}+\sqrt{1-\boldsymbol{v}_{1}^{2}}\right)\left(\sqrt{1-\boldsymbol{v}_{1}^{2}}-1\right)}
$$

whereby $\Lambda_{k}^{i}(\boldsymbol{\nu} ; \boldsymbol{v})=\Lambda_{j}^{i}\left(\boldsymbol{\nu} ; \boldsymbol{v}_{2}\right) \Lambda_{k}^{j}\left(\boldsymbol{\nu} ; \boldsymbol{v}_{1}\right)$. It is thus clear that equation (10) represents the Einstein law of addition of velocities $\boldsymbol{v}_{1}$ and $\boldsymbol{v}_{2}$. In comparison with its usual form one should remember, however, that after the transformation $\Lambda_{k}^{j}\left(\boldsymbol{\nu} ; \boldsymbol{v}_{1}\right)$ the spatial axes, in which the $\boldsymbol{v}_{2}$ is prescribed, appear now to be not parallel to the initial axes, but turned so that the vector $\boldsymbol{\nu}$ relative to them maintains its initial orientation. Therefore, it is true, irrespective of the direction of $\boldsymbol{v}_{1}$, equation (10) yields $\boldsymbol{v}=\boldsymbol{\nu}$ if $\boldsymbol{v}_{2}=\boldsymbol{\nu}$.

\subsection{3-parameter group of the generalized Lorentz transformations}

The 3-parameter group of the generalized Lorentz transformations (of the generalized Lorentz boosts), similarly to the subgroup (2) of Lorentz group, consists of noncompact transformations only. In the infinitesimal form the transformations belonging to it appear as

$$
\begin{aligned}
& d x^{0}=\left(-r(\boldsymbol{\nu} n) x^{0}-\boldsymbol{n x}\right) d \alpha, \\
& d \boldsymbol{x}=\left(-r(\boldsymbol{\nu n}) \boldsymbol{x}-\boldsymbol{n} x^{0}-[\boldsymbol{x}[\boldsymbol{\nu n}]]\right) d \alpha .
\end{aligned}
$$

Here, as in the infinitesimal transformations (1) of the group (2), the $\boldsymbol{n}$ and $\alpha$ are group parameters, while the $\boldsymbol{\nu}$ is a fixed unit vector. And the difference between (11) and (1) consists in the appearance of an additional generator of the scale transformations, which is proportional to a new fixed dimensionless parameter $r$. Since the scale transformations commute with the Lorentz boosts and 3D rotations, the result of integration of equations (11) is a priori clear:

$$
x^{i}=D(r, \boldsymbol{\nu} ; \boldsymbol{n}, \alpha) \Lambda_{k}^{i}(\boldsymbol{\nu} ; \boldsymbol{n}, \alpha) x^{k},
$$

where $D(r, \boldsymbol{\nu} ; \boldsymbol{n}, \alpha)=e^{-r \boldsymbol{\nu} \boldsymbol{n} \alpha} I$, whereby $I$ is a unit matrix while $\Lambda_{k}^{i}(\boldsymbol{\nu} ; \boldsymbol{n}, \alpha)$ are matrices which make up the group (2). As for the law of group composition for the group (12), it is given by equations (5) and (6) obtained for the group (2). We note incidentally that equation (5) yields the relation $\boldsymbol{\nu} \boldsymbol{n} \alpha=\boldsymbol{\nu} \boldsymbol{n}_{1} \alpha_{1}+\boldsymbol{\nu} \boldsymbol{n}_{2} \alpha_{2}$. Thus the group of the generalized Lorentz transformations (12), on the one hand, is locally isomorphic to the corresponding 3-parameter subgroup (2) of the Lorentz group and, on the other hand, it is a homogeneous 3-parameter noncompact subgroup of the similitude group [13]. Since (according to (12)) in passing to the primed inertial frame the time $\left(x^{0}\right)$ and space $(\boldsymbol{x})$ event coordinates undergo identical additional dilatations $D$, then the velocity $\boldsymbol{v}$ of the primed frame is related to the group parameters $\boldsymbol{n}, \alpha$ by the same equations (7)(9) as in the case $r=0$ where the group (12) coincides with the subgroup (2) of the Lorentz group. For the same reason the transformations (12) retain valid Einstein's law of addition of 3 -velocities, written as (10). As for the reparametrization of the group (12), then, for example, the matrix $D$, involved in (12), takes the following form in terms of $\boldsymbol{v}$ :

$$
D(r, \boldsymbol{\nu} ; \boldsymbol{v})=\left(\frac{1-\boldsymbol{v} \boldsymbol{\nu}}{\sqrt{1-\boldsymbol{v}^{2}}}\right)^{r} I
$$

Unlike the transformations (2), the 3-parameter group of the generalized Lorentz boosts (12) conformally modifies the Minkowski metric but leaves invariant the metric

$$
d s^{2}=\left[\frac{\left(d x_{0}-\boldsymbol{\nu} d \boldsymbol{x}\right)^{2}}{d x_{0}^{2}-d \boldsymbol{x}^{2}}\right]^{r}\left(d x_{0}^{2}-d \boldsymbol{x}^{2}\right) .
$$


The given Finslerian metric generalizes the Minkowski metric and describes the relativistically invariant flat space of events with partially broken 3D isotropy. The inhomogeneous isometry group of space (14) is an 8-parameter one: four parameters correspond to space-time translations, one parameter, to rotations about the physically preferred direction $\boldsymbol{\nu}$, and three parameters, to the generalized Lorentz boosts.

\subsection{Bispinor representation of the 3-parameter group of generalized Lorentz boosts}

Now turn to the construction of bispinor representation of the group of the generalized Lorentz boosts (12). Since the group (12) is locally isomorphic to the 3-parameter subgroup (2) of the Lorentz group, its bispinor representation must also be locally isomorphic to the bispinor representation of the subgroup (2). This means that the transformations $x^{i}=D \Lambda_{k}^{i} x^{k}$ of the event coordinates should be accompanied by the following transformations of a bispinor field: $\Psi^{\prime}\left(x^{\prime}\right)=D^{d} S \Psi(x), \bar{\Psi}^{\prime}\left(x^{\prime}\right)=\bar{\Psi}(x) D^{d} S^{-1}$. Here the group of matrices $S$, operating on the bispinor indices, represents the subgroup (2) of the Lorentz matrices $\Lambda_{k}^{i}$ while $D^{d}$ denotes the corresponding additional scale transformations of bispinors, in which case the unit matrix, involved in the definition (13), operates on the bispinor indices in this context. Since $d^{4} x^{\prime}=\left|D \Lambda_{k}^{i}\right| d^{4} x=D^{4} d^{4} x$ and matrices $S$ satisfy the standard condition $S^{-1} \gamma^{n} S=\Lambda_{m}^{n} \gamma^{m}$, then, proceeding from the generalized Lorentz invariance of action for a free massless field $\Psi$, it is easy to show that $d=-3 / 2$. As a result the bispinor representation of the group of generalized Lorentz boosts (12) is realized by the transformations

$$
\Psi^{\prime}=D^{-3 / 2} S \Psi, \quad \bar{\Psi}^{\prime}=\bar{\Psi} D^{-3 / 2} S^{-1}
$$

and it remains to find a 3-parameter group of the matrices $S=S(\boldsymbol{\nu} ; \boldsymbol{n}, \alpha)$. For this purpose, using the 4-vectors $\nu^{l}=(1, \boldsymbol{\nu}) ; \nu_{l}=(1,-\boldsymbol{\nu}) ; n^{l}=(0, \boldsymbol{n}) ; n_{l}=(0,-\boldsymbol{n})$, first rewrite the infinitesimal transformations (1) in the form $d x^{i}=\omega_{k}^{i} x^{k}$, where $\omega_{k}^{i}=\left(\nu^{i} n_{k}-n^{i} \nu_{k}\right) d \alpha$, in which case $-\omega_{k i}=\omega_{i k}=\left(\nu_{i} n_{k}-\nu_{k} n_{i}\right) d \alpha$. Thus, in the vicinity of the identical transformation the matrices $\Lambda_{k}^{i}$ take the form $\Lambda_{k}^{i}(d \alpha)=\delta_{k}^{i}+\omega^{i}{ }_{k}$. Respectively, the $S(d \alpha)=1+\frac{1}{8}\left(\gamma^{i} \gamma^{k}-\gamma^{k} \gamma^{i}\right) \omega_{i k}$. Considering that $n_{0}=0$ and $\nu_{0}=1$, the latter relation leads to $S$ in the form:

$$
S(\boldsymbol{\nu} ; \boldsymbol{n}, \alpha)=e^{\{\cdots\} \alpha / 2} .
$$

Here and below we use the notation $\{\cdots\}$ for the sum of generators of Lorentz boosts and 3 -rotations about the vector $[\boldsymbol{\nu n}]$, that is

$$
\{\cdots\}=-\gamma^{0} \gamma \boldsymbol{n}-i \boldsymbol{\Sigma}[\boldsymbol{\nu} \boldsymbol{n}]
$$

where $\gamma^{0}, \gamma$ are the Dirac matrices, $\boldsymbol{\Sigma}=\operatorname{diag}(\boldsymbol{\sigma}, \boldsymbol{\sigma})$ and $\boldsymbol{\sigma}$ are the Pauli matrices. With the aid of the algebra of $\gamma$ matrices one can find that

$$
\begin{array}{lll}
\{\cdots\}^{2}=(\boldsymbol{\nu n})^{2} I, & \{\cdots\}^{4}=(\boldsymbol{\nu} \boldsymbol{n})^{4} I, & \cdots ; \\
\{\cdots\}^{3}=(\boldsymbol{\nu n})^{3}\{\cdots\} /(\boldsymbol{\nu n}), & \{\cdots\}^{5}=(\boldsymbol{\nu n})^{5}\{\cdots\} /(\boldsymbol{\nu n}), & \cdots
\end{array}
$$

These relations make it possible to represent (16) as

$$
S(\boldsymbol{\nu} ; \boldsymbol{n}, \alpha)=I \cosh \frac{\boldsymbol{\nu} \boldsymbol{n} \alpha}{2}-\frac{i \boldsymbol{\Sigma}[\boldsymbol{\nu} \boldsymbol{n}]+\gamma^{0} \boldsymbol{\gamma} \boldsymbol{n}}{\boldsymbol{\nu} \boldsymbol{n}} \sinh \frac{\boldsymbol{\nu} \boldsymbol{n} \alpha}{2} .
$$

Now reparametrizing the $S(\boldsymbol{\nu} ; \boldsymbol{n}, \alpha)$ with the aid of (8), (9) and using equations (15) and (13), we thus arrive at the following 3-parameter noncompact group of bispinor transformations in 
the axially symmetric flat Finslerian space (14):

$$
\begin{aligned}
\Psi^{\prime}= & \frac{\left((1-\boldsymbol{v} \boldsymbol{\nu}) / \sqrt{1-\boldsymbol{v}^{2}}\right)^{-3 r / 2}}{2 \sqrt{(1-\boldsymbol{v} \boldsymbol{\nu}) \sqrt{1-\boldsymbol{v}^{2}}}}\left\{\left(1-\boldsymbol{v} \boldsymbol{\nu}+\sqrt{1-\boldsymbol{v}^{2}}\right) I\right. \\
& \left.-i[\boldsymbol{\nu} \boldsymbol{v}] \boldsymbol{\Sigma}-\left(\boldsymbol{v}-\left(1-\sqrt{1-\boldsymbol{v}^{2}}\right) \boldsymbol{\nu}\right) \gamma^{0} \gamma\right\} \Psi .
\end{aligned}
$$

We note finally that an invariant of the transformations (19) is the Finslerian form

$$
\left[\left(\nu_{n} \bar{\Psi} \gamma^{n} \Psi / \bar{\Psi} \Psi\right)^{2}\right]^{-3 r / 2} \bar{\Psi} \Psi
$$

but no longer the bilinear form $\bar{\Psi} \Psi$.

\section{Finite transformations belonging to two subgroups of the 3-parameter group of generalized Lorentz boosts}

\subsection{Abelian 2-parameter subgroup and its geometric invariants}

The Abelian 2-parameter subgroup is generated by the infinitesimal transformations (11) provided that $\boldsymbol{n} \perp \boldsymbol{\nu}$, and its finite transformations can be derived from the finite 3-parameter transformations (12) via the passage to the limit $(\boldsymbol{\nu} \boldsymbol{n} \alpha) \rightarrow 0$. As a result, we find

$$
\begin{aligned}
& x_{0}^{\prime}=\left(\frac{\alpha^{2}}{2}+1\right) x_{0}-\alpha(\boldsymbol{n x})-\frac{\alpha^{2}}{2}(\boldsymbol{\nu} \boldsymbol{x}), \\
& \boldsymbol{x}^{\prime}=\boldsymbol{x}+\boldsymbol{n}\left(-x_{0}+\boldsymbol{\nu} \boldsymbol{x}\right) \alpha+\boldsymbol{\nu}\left[\left(x_{0}-\boldsymbol{\nu} \boldsymbol{x}\right) \frac{\alpha^{2}}{2}-(\boldsymbol{n x}) \alpha\right] .
\end{aligned}
$$

The inverse transformations are obtained by means of the substitution $\alpha \rightarrow-\alpha$ :

$$
\begin{aligned}
& x_{0}=\left(\frac{\alpha^{2}}{2}+1\right) x_{0}^{\prime}+\alpha\left(\boldsymbol{n} \boldsymbol{x}^{\prime}\right)-\frac{\alpha^{2}}{2}\left(\boldsymbol{\nu} \boldsymbol{x}^{\prime}\right), \\
& \boldsymbol{x}=\boldsymbol{x}^{\prime}+\boldsymbol{n}\left(x_{0}^{\prime}-\boldsymbol{\nu} \boldsymbol{x}^{\prime}\right) \alpha+\boldsymbol{\nu}\left[\left(x_{0}^{\prime}-\boldsymbol{\nu} \boldsymbol{x}^{\prime}\right) \frac{\alpha^{2}}{2}+\left(\boldsymbol{n} \boldsymbol{x}^{\prime}\right) \alpha\right] .
\end{aligned}
$$

As before, in order to express the velocity $\boldsymbol{v}$ of the primed inertial frame in terms of the parameters $\boldsymbol{n}, \alpha$ it is sufficient to consider in the initial frame, the motion of the origin of the primed frame, i.e. to put $\boldsymbol{x}^{\prime}=0$. Then we get $x_{0}=\left(\alpha^{2} / 2+1\right) x_{0}^{\prime}, \boldsymbol{x}=\left(\boldsymbol{n} \alpha+\boldsymbol{\nu} \alpha^{2} / 2\right) x_{0}^{\prime}$. As a result,

$$
\boldsymbol{v}=\frac{\boldsymbol{n} \alpha+\boldsymbol{\nu} \alpha^{2} / 2}{1+\alpha^{2} / 2}
$$

It is easy to verify that in terms of $\boldsymbol{v}$ the condition $\boldsymbol{n} \perp \boldsymbol{\nu}$ takes the form

$$
\left(\frac{1-\boldsymbol{v} \boldsymbol{\nu}}{\sqrt{1-\boldsymbol{v}^{2}}}\right)^{2}=1
$$

Thus, having used the equation (22) for reparametrization of the initial transformations (20), we get

$$
\begin{aligned}
& x_{0}^{\prime}=\frac{x_{0}-\boldsymbol{v} \boldsymbol{x}}{1-\boldsymbol{v} \boldsymbol{\nu}}, \\
& \boldsymbol{x}^{\prime}=\boldsymbol{x}-\frac{\left(x_{0}-\boldsymbol{\nu} \boldsymbol{x}\right) \boldsymbol{v}-\left[\left(2 x_{0}-\boldsymbol{\nu} \boldsymbol{x}\right)(\boldsymbol{v} \boldsymbol{\nu})-(\boldsymbol{v} \boldsymbol{x})\right] \boldsymbol{\nu}}{1-\boldsymbol{v} \boldsymbol{\nu}} .
\end{aligned}
$$


One can verify that these transformations have two independent invariants: $\left(x_{0}^{2}-\boldsymbol{x}^{2}\right)$ and $\left(x_{0}-\boldsymbol{\nu} \boldsymbol{x}\right)$. Therefore the transformations (23) belong to the 6-parameter Lorentz group. At the same time, leaving the Finslerian metric (14) invariant, they make up the 2-parameter Abelian subgroup of the 3-parameter group of generalized Lorentz boosts (12).

In spite of a new ( Finslerian ) geometry of the event space the 3-velocity space remains the Lobachevski space. This means that the generalized Lorentz boosts (12) induce the corresponding motions of the Lobachevski space. In particular, the above-described Abelian 2-parameter boosts (23) induce such motions which leave invariant a family of the horospheres

$$
\frac{1-\boldsymbol{v} \nu}{\sqrt{1-v^{2}}}=\text { const }
$$

i.e. of surfaces perpendicular to the congruence of geodesics parallel to $\boldsymbol{\nu}$ and possessing Euclidean inner geometry.

\subsection{1-parameter subgroup and its geometric invariants}

Finite transformations, making up the 1-parameter subgroup of the 3-parameter group of generalized Lorentz boosts, follow from the 3-parameter finite transformations (12) provided that $\boldsymbol{n} \| \boldsymbol{\nu}$. As a result we get

$$
\begin{aligned}
& x_{0}^{\prime}=e^{-r \alpha}\left\{x_{0} \cosh \alpha-(\boldsymbol{\nu} \boldsymbol{x}) \sinh \alpha\right\}, \\
& \boldsymbol{x}^{\prime}=e^{-r \alpha}\left\{\boldsymbol{x}-\boldsymbol{\nu}(\boldsymbol{\nu} \boldsymbol{x})+\boldsymbol{\nu}\left[-x_{0} \sinh \alpha+(\boldsymbol{\nu} \boldsymbol{x}) \cosh \alpha\right]\right\} .
\end{aligned}
$$

The inverse 1-parameter transformations have the form

$$
\begin{aligned}
& x_{0}=e^{r \alpha}\left\{x_{0}^{\prime} \cosh \alpha+\left(\boldsymbol{\nu} \boldsymbol{x}^{\prime}\right) \sinh \alpha\right\}, \\
& \boldsymbol{x}=e^{r \alpha}\left\{\boldsymbol{x}^{\prime}-\boldsymbol{\nu}\left(\boldsymbol{\nu} \boldsymbol{x}^{\prime}\right)+\boldsymbol{\nu}\left[x_{0}^{\prime} \sinh \alpha+\left(\boldsymbol{\nu} \boldsymbol{x}^{\prime}\right) \cosh \alpha\right]\right\} .
\end{aligned}
$$

According to equations (25), the velocity $\boldsymbol{v}$ of the primed system is related to the $\boldsymbol{\nu}$ and the group parameter $\alpha$ by the relations

$$
\begin{aligned}
& \boldsymbol{v}=\boldsymbol{\nu} \tanh \alpha, \\
& \frac{\boldsymbol{v} \boldsymbol{\nu}}{\sqrt{1-\boldsymbol{v}^{2}}}=\sinh \alpha, \quad \frac{1}{\sqrt{1-\boldsymbol{v}^{2}}}=\cosh \alpha, \quad \frac{1-\boldsymbol{v} \boldsymbol{\nu}}{\sqrt{1-\boldsymbol{v}^{2}}}=e^{-\alpha} .
\end{aligned}
$$

Since

$$
\begin{aligned}
& \left(x_{0}^{\prime}-\boldsymbol{\nu} \boldsymbol{x}^{\prime}\right)=e^{(1-r) \alpha}\left(x_{0}-\boldsymbol{\nu} \boldsymbol{x}\right), \\
& \left(x_{0}^{\prime 2}-\boldsymbol{x}^{\prime 2}\right)=e^{-2 r \alpha}\left(x_{0}^{2}-\boldsymbol{x}^{2}\right), \\
& {\left[\boldsymbol{x}^{\prime} \boldsymbol{\nu}\right]=e^{-r \alpha}[\boldsymbol{x} \boldsymbol{\nu}]}
\end{aligned}
$$

we see that the 1-parameter group of generalized Lorentz boosts (25) conformally modifies the Minkowski metric, but leaves invariant the Finslerian metric (14). In addition, we get the following invariant of this group:

$$
\frac{[\boldsymbol{x} \boldsymbol{\nu}]}{\sqrt{x_{0}^{2}-\boldsymbol{x}^{2}}}=\text { invar. }
$$

In the Lobachevski space of the 3-velocities $\boldsymbol{v}$ the 1-parameter transformations under consideration induce such motions which leave invariant a family of the surfaces

$$
\frac{\boldsymbol{v}^{2}-(\boldsymbol{v} \boldsymbol{\nu})^{2}}{1-\boldsymbol{v}^{2}}=\text { const. }
$$

These surfaces have the form of cylinders formed by the rotation of the equidistant lines about $\boldsymbol{\nu}$. 


\section{Conclusion}

Within the framework of the Finslerian approach to the problem of violation of the Lorentz symmetry we have studied the flat axially symmetric Finslerian space of events which is a generalization of Minkowski space. Such event space arises from spontaneous breaking of initial gauge symmetry and from formation of an anisotropic fermion-antifermion condensate. We have shown that the appearance of an anisotropic condensate breaks the Lorentz symmetry but the relativistic symmetry, realized by means of the 3-parameter group of generalized Lorentz boosts, remains valid here. The principle of generalized Lorentz invariance enables exact taking into account the influence of an axially symmetric condensate on the dynamics of fundamental fields. In particular, with its aid in [14], the Lagrangian, describing the dynamics of the massive fermion field in the relativistically invariant anisotropic condensate, has been constructed. This Lagrangian leads to some nonlinear spinor equations. The algebraic-theoretical methods of constructing exact solutions for a wide class of nonlinear spinor equations, developed by W.I. Fushchych with coworkers [15], make it possible to find the so-called nongenerable solutions of the above-mentioned spinor equations. Just with this objective in view the subgroups of the group of generalized Lorentz transformations and their geometric invariants have been studied in the present work.

\section{Acknowledgements}

The author is grateful to Prof. H. Goenner for the fruitful collaboration that led to the results presented in this paper. It is pleasure to thank also Prof. R. Tavakol for helpful discussions.

[1] Kostelecký A., Samuel S., Spontaneous breaking of Lorentz symmetry in string theory, Phys. Rev. D, 1989, V.39, N 2, 683-685.

[2] Colladay D., Kostelecký A., CPT violation and the standard model, Phys. Rev. D, 1997, V.55, N 11, 67606774; hep-ph/9703464.

Colladay D., Kostelecký A., Lorentz-violating extension of the standard model, Phys. Rev. D, 1998, V.58, 116002; hep-ph/9809521.

[3] Kostelecký A., Gravity, Lorentz violation, and the standard model, Phys. Rev. D, 2004, V.69, 105009; hep-th/0312310.

[4] Kostelecký A. (Editor), CPT and Lorentz symmetry III, Singapore, World Scientific, 2005.

[5] Allen R.E., Yokoo S., Searching for Lorentz violation, Nucl. Phys. Proc. Suppl. B, 2004, V.134, 139-146; hep-th/0402154.

[6] Cardone F., Mignani R., Broken Lorentz invariance and metric description of interactions in a deformed Minkowski space, Found. Phys., 1999, V.29, N 11, 1735-1783.

[7] Bogoslovsky G.Yu., A special relativistic theory of the locally anisotropic space-time, Nuovo Cim. B, 1977, V.40, N 1, 99-134.

[8] Bogoslovsky G.Yu., Theory of locally anisotropic space-time, Moscow, Moscow Univ. Press, 1992.

[9] Bogoslovsky G.Yu., From the Weyl theory to a theory of locally anisotropic space-time, Class. Quantum Grav., 1992, V.9, 569-575.

Bogoslovsky G.Yu., Finsler model of space-time, Phys. Part. Nucl., 1993, V.24, 354-379.

Bogoslovsky G.Yu., A viable model of locally anisotropic space-time and the Finslerian generalization of the relativity theory, Fortschr. Phys., 1994, V.42, N 2, 143-193.

[10] Bogoslovsky G.Yu., Goenner H.F., On the possibility of phase transitions in the geometric structure of space-time, Phys. Lett. A, 1998, V.244, 222-228; gr-qc/9804082.

Bogoslovsky G.Yu., Goenner H.F., Finslerian spaces possessing local relativistic symmetry, Gen. Rel. Grav., 1999, V.31, N 10, 1565-1603; gr-qc/9904081.

[11] Arbuzov B.A., Muon g-2 anomaly and extra interaction of composite Higgs in a dynamically broken electroweak theory, hep-ph/0110389.

Arbuzov B.A., Spontaneous generation of effective interaction in a renormalizable quantum field theory model, Teor. Mat. Fiz., 2004, V.140, N 3, 367-387 (and references therein). 
[12] Winternitz P., Friš I., Invariant expansions of relativistic amplitudes and subgroups of the proper Lorentz group, Yadern. Fiz., 1965, V.1, N 5, 889-901.

[13] Patera J., Winternitz P., Zassenhaus H., Continuous subgroups of the fundamental groups of physics. II. The similitude group, J. Math. Phys., 1975, V.16, N 8, 1615-1624.

[14] Bogoslovsky G.Yu., Goenner H.F., On the generalization of the fundamental field equations for locally anisotropic space-time, in Proceedings of XXIV International Workshop "Fundamental Problems of High Energy Physics and Field Theory" (June 27-29, 2001, Protvino, Russia), Editor V.A. Petrov, Protvino, Insitute for High Energy Physics, 2001, 113-125.

Bogoslovsky G.Yu., Goenner H.F., Concerning the generalized Lorentz symmetry and the generalization of the Dirac equation, Phys. Lett. A, 2004, V.323, 40-47; hep-th/0402172.

[15] Fushchych W.I., Zhdanov R.Z., Symmetry and exact solutions of nonlinear spinor equations, Phys. Rep., 1989, V.172, N 4, 123-174.

Fushchych W.I., Zhdanov R.Z., Symmetry and exact solutions of nonlinear Dirac equations, Kyiv, Mathematical Ukraina Publisher, 1997 (and references therein). 